\title{
Hydrogen in subsurface sites of Pd(111): Self-consistent electronic structure
}

\author{
C. T. Chan and Steven G. Louie \\ Department of Physics, University of California, Berkeley, California 94720 \\ and Lawrence Berkeley Laboratory, Berkeley, California 94720 \\ (Received 20 April 1984)
}

\begin{abstract}
The electronic structures of monolayer atomic hydrogen in subsurface sites of the Pd(111) surface are studied with self-consistent pseudopotential calculations. Results of the present calculations include the density of states, electronic character of the $\mathbf{P d}-\mathbf{H}$ bond, and analyses of surface states and surface density of states. It is shown that $\mathbf{H}$ in subsurface tetrahedral sites have electronic properties very similar to those of $\mathbf{H}$ in the surface threefold sites. On the other hand, near the zone center, the electronic properties for $\mathbf{H}$ in subsurface octahedral sites are similar to the clean surface. This is a consequence of the symmetry of the (111) surface. The H-induced features for this geometry would then be difficult to detect by normal-emission spectroscopy, which samples states near the zone center, but should be observable at the zone boundaries by angle-resolved photoemission spectroscopy. The subsurface chemisorption model for the high-temperature phase of $\mathrm{H} / \mathrm{Pd}(111)$ is discussed in light of the present calculation.
\end{abstract}

\section{INTRODUCTION}

Chemisorption on transition-metal surfaces has been actively studied both theoretically and experimentally in the past decade. ${ }^{1}$ There are abundant experimental data on these systems but reliable theoretical calculations are comparatively few due to the difficulty of treating the $d$-like electrons in transition metals. However, in recent years, advances in theory have furnished us with more realistic calculations, ${ }^{1-5}$ and certain aspects of photoemission experiments have been successfully interpreted. Various theoretical calculations have demonstrated that the adsorbate-induced features are sensitive to the local geometry of chemisorption. ${ }^{2,3}$

In the particular case of atomic $\mathbf{H}$ on fcc group-VIII transition-metal surfaces, conspicuous changes are induced in the photoemission spectra upon chemisorption. ${ }^{6}$ These include quenched intrinsic surface states and a split-off hydrogen-induced band. By comparing experimental results with theoretical calculations, useful information can be obtained about the site of $\mathrm{H}$ chemisorption and the nature of the chemisorption bond. For example, such an approach has been applied successfully previously to a low-temperature phase of $\mathbf{H}$ on $\mathrm{Pd}(111)$. Comparison of angle-integrated ${ }^{2}$ and angle-resolved ${ }^{7}$ photoemission data with the corresponding theoretically determined spectra has revealed that at low temperature $(<250 \mathrm{~K}), \mathrm{H}$ occupies the surface threefold sites and forms a $(1 \times 1)$ ordered layer on the Pd(111) surface.

Recent angle-resolved photoemission experiments further showed that the $\mathrm{H}$-induced features are temperature dependent. ${ }^{6,7}$ At room temperature, all the $\mathrm{H}$-induced features disappear with a normal-emission spectrum very similar to the clean surface, although experimental evidence indicates that $H$ still resides at or near the $\operatorname{Pd}(111)$ surface in substantial amount. ${ }^{6,7}$ The same phenomenon has also been observed on $\mathrm{Ni}(111), \mathrm{Pt}(111),{ }^{6}$ and recently on $\mathrm{Cu}(111){ }^{8}$ This is in contradiction to previous results, as the $\operatorname{Pd}(111)$ intrinsic surface states are known to be sensitive to adsorbates, ${ }^{2}$ and the $\mathrm{H}$-induced split-off band is observed experimentally upon the formation of $\mathrm{Pd}-\mathrm{H}$ bonding in all other situations. ${ }^{9}$ To explain this peculiar feature, Eberhardt et al. have proposed ${ }^{6}$ that the $\mathrm{H}$ might migrate from surface threefold sites to the subsurface sites upon warming. The calculations of Feibelman et al. ${ }^{3}$ did show that the $d$-like surface states are not strongly perturbed by $\mathrm{H}$ in the subsurface sites of $\mathrm{Ti}(0001)$. However, this conjecture has one obvious difficulty. It cannot easily explain the disappearance of the $\mathrm{H}$-induced state. It is well established that a $\mathrm{H}$-induced split-off band exists even for dilute palladium hydrides. ${ }^{10}$ In fact, Klein and Pickett $^{11}$ have shown that even for a single-H impurity in $\mathrm{Pd}$ octahedral sites, such a $\mathrm{H}$-induced peak appears in the local density of states. Thus the subsurface model is valid only if the $\mathbf{P d}-\mathbf{H}$ bondings for $\mathbf{H}$ in the subsurface sites have very different electronic characters as compared to $\mathbf{H}$ on surface or in bulk sites.

Recent theoretical efforts in chemisorption are mostly devoted to the study of surface or bulk electronic properties. Properties of the subsurface sites should be worth equal attention, since they are in a local environment somewhere between the surface and the bulk sites, and these are the sites through which the adsorbates enter the bulk. In view of the above experimental results for the $\mathrm{H} / \mathrm{Pd}(111)$ system, it is of particular interest to determine whether $\mathbf{H}$ induces a split-off bonding band in subsurface chemisorption geometries and to examine whether the $\mathrm{Pd}$ surface states are perturbed. There is rich literature for the electronic structure of bulk Pd hydrides. ${ }^{10,12}$ The electronic structures of $\mathbf{H}$ in surface top sites, surface threefold sites, ${ }^{2,7}$ and bulk octahedral sites ${ }^{12}$ have been treated successfully by the mixed-basis pseudopotential method. It is the purpose of the present work to calculate the electronic structure of $\mathbf{H}$ chemisorbed in subsurface sites and compare to that of the surface sites and bulk sites. We found that for $\mathbf{H}$ in both subsurface tetrahedral and octa- 
hedral sites a well-separated $\mathrm{H}$-induced band exists. This induced band is due to bonding between the $\mathrm{Pd} 4 d$ and $\mathrm{H}$ $1 s$ orbitals, as in the case of $\mathrm{H}$ on surface threefold and in bulk sites. However, near the center of the twodimensional Brillouin zone (2D BZ) the $\mathrm{Pd}-\mathrm{H}$ bonding is $s-s$ for $\mathbf{H}$ in the octahedral site, as opposed to mainly $s-d$ for other surface and subsurface sites. This bonding character, a consequence of the symmetry of the fcc (111) surface, may render the $\mathrm{H}$-induced peak invisible in normalemission experiments since $s$-like states have a weaker cross section compared with $d$-like states. ${ }^{13}$ We also found that the surface states and resonances of $\mathrm{Pd}(111)$ surface are in general less strongly perturbed for $\mathrm{H}$ in the octahedral sites than in other sites.

The remainder of the paper is organized as follows. The method of calculation is described in Sec. II. The results are presented in Sec. III. Section IV presents the discussion, and Sec. $\mathrm{V}$ is a summary.

\section{CALCLLATIONS}

The calculations were performed using the fully selfconsistent mixed-basis pseudopotential method ${ }^{14}$ within the local-density approximation. The Hedin-Lundqvist ${ }^{15}$ local exchange-correlation potential was used. The input to our calculations was the atomic geometries and the ionic pseudopotentials. The ionic pseudopotentials have been reported elsewhere. ${ }^{12}$ The surface problem was treated by a supercell description. The supercell was comprised of a slab of seven layers of $\mathrm{Pd}$ and a vacuum region equivalent to $4 \mathrm{Pd}$ layers. A monolayer of $\mathrm{H}$ atoms is placed in the appropriate sites on both sides of the $\mathrm{Pd}$ slab. The clean $\operatorname{Pd}(111)$ surface has $C_{3 v}$ symmetry. (The slab geometry used in our calculations has, in addition, an inversion center.) Chemisorption of $\mathbf{H}$ on the high-symmetry sites we considered preserves this symmetry. The group theory for the fcc (111) surface has been treated by Caruthers et al. ${ }^{16}$

The mixed-basis set used in expanding the wave function consists of both plane waves and Gaussians. This combination offers a flexible and efficient basis set, which can treat both localized and delocalized electrons simultaneously and is thus very suitable for transition-metal compounds. The present method has been successfully applied to palladium-hydrogen systems ${ }^{2,12}$ and other transition-metal systems. ${ }^{14}$ In the present calculations, the wave function is expanded in $\sim 325$ plane waves and a localized set of 35 Gaussians in Bloch form (five for each $\mathbf{P d}$ atom in the unit cell). The calculations are regarded as self-consistent if the Fourier components of the input and output potentials have a maximum difference of less than $0.05 \mathrm{Ry}$. The eigenvalues are converged to at least one order of magnitude better than the potential.

The $\mathbf{H}$ chemisorption sites considered in the present work are the subsurface octahedral and tetrahedral sites. Results for $\mathbf{H}$ in the surface threefold sites ${ }^{2}$ and the clean (111) surface $^{7}$ are also used for comparison. There are two types of surface threefold sites on the (111) surface, depending on whether there is a second layer of Pd directly below the $\mathbf{H}$ atom (hcp site) or not (fcc site). Since the $\mathbf{P d}-\mathbf{H}$ bonding is short ranged and hardly extends beyond the nearest neighbors, chemisorption on these two sites yields very similar electronic properties. ${ }^{2}$ Muscat $^{18}$ found that this is true for most of the $3 d$ and $4 d$ fcc transition metals. Thus, in the present paper, we shall only compare results with those of the hcp site. The octahedral and tetrahedral sites are high-symmetry sites with six and four nearest-neighbor $\mathbf{P d}$ atoms, respectively. Octahedral sites are occupied by $\mathbf{H}$ in the $\beta$-phase bulk Pd hydride. Figure 1 shows the position of these sites on a (110) plane.

For $\mathrm{H}$ on the surface threefold sites, the Pd layers are assumed to be unrelaxed and unreconstructed. The $\mathrm{Pd}-\mathrm{H}$ bond length is taken to be the sum of metallic radius of $P d$ and covalent radius of $H$. This assumption is justified by the excellent agreement between calculated and experimental angle-resolved photoemission spectra for the lowtemperature phase. ${ }^{7}$ For $\mathbf{H}$ at the subsurface sites, the topmost layer of Pd is relaxed outward by $2 \%$, as suggested by the LEED experiments. ${ }^{19}$ The position of $\mathrm{H}$ is fixed by symmetry.

In the present calculations, the $\mathbf{H}$ at the subsurface tetrahedral site is actually in an environment rather similar to the $\mathbf{H}$ at surface threefold sites. At the subsurface tetrahedral sites, $\mathbf{H}$ has four nearest-neighbor $\mathbf{P d}$, of which three are on the top layer. The $\mathbf{P d}-\mathbf{H}$ bond length and bond angle between the chemisorbed $\mathrm{H}$ and its three nearest-neighbor surface $\mathbf{P d}$ atoms are practically the same for the surface threefold sites and the subsurface tetrahedral sites. The only difference is that $\mathrm{H}$ at the subsurface tetrahedral sites has an extra nearest-neighbor $\mathbf{P d}$ directly below it. We thus expect qualitative similarity in the electronic structure of these two systems. On the oth-

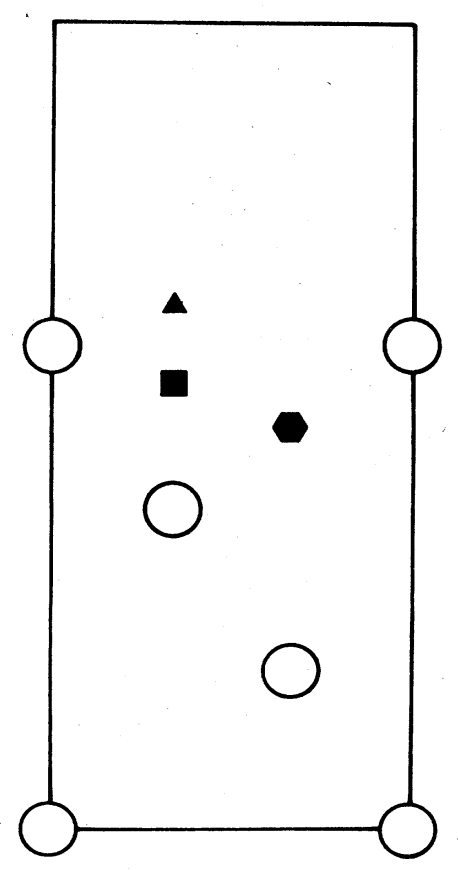

FIG. 1. Positions of the surface threefold site (triangle), the subsurface tetrahedral site (square), and the subsurface octahedral site (hexagon) on a (110) plane. The open circles are the Pd atoms. 
er hand, $\mathrm{H}$ at the octahedral sites has six nearest-neighbor Pd. The Pd-H bond length is longer, while the bond angle is smaller as compared to that of subsurface tetrahedral sites.

\section{RESULTS}

\section{A. Density of states}

The total density of states (DOS) of the slab for $\mathbf{H}$ at surface threefold and subsurface octahedral and tetrahedral sites are plotted in Fig. 2. Energy eigenvalues at a regular mesh of 15 points in the $2 \mathrm{D} \mathrm{BZ}$ are calculated from first principles for each of the systems and are then fitted band by band to a symmetrized Fourier function with ten coefficients. The DOS are then determined from the eigenvalues generated by the Fourier fit. Such a scheme cannot take into account band crossings at symmetry lines and points but should be accurate for integrated features like DOS. The important features to note are

(1) A H-induced band exists and splits off from the Pd $d$ bands for all three cases. A similar split-off band is well known in the bulk hydride. For $\mathbf{H}$ on surface threefold sites, it is peaked at $-6.5 \mathrm{eV}$, in very good agreement

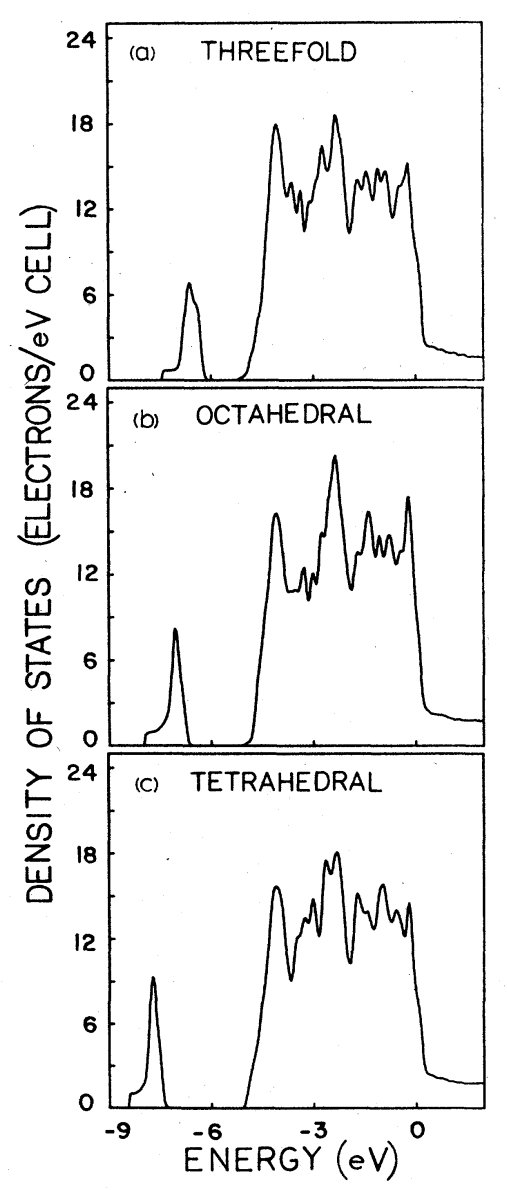

FIG. 2. Total density of states for $\mathbf{H}$ on $\mathbf{P d}(111)$ (seven layers of $\mathbf{P d}$ and two layers of $\mathbf{H}$ ) at various sites. The Fermi energy is at $0 \mathrm{eV}$. with that observed in angle-integrated photoemission experiments. ${ }^{6,20}$

(2) The $\mathrm{H}$-induced peak is lower in energy for $\mathrm{H}$ at the subsurface sites than for $\mathrm{H}$ at surface threefold sites. Between subsurface sites, it is lower for the tetrahedral sites than the octahedral sites. This is consistent with the fact that the subsurface sites have a higher coordination number than the surface sites and that the Pd-H bond length is shorter for the tetrahedral sites than the octahedral sites. We also note that the $\mathbf{P d}-\mathrm{H}$ bonding band has the highest energy for $\mathbf{H}$ on the surface top sites, ${ }^{2}$ which has the smallest coordination number.

(3) Near the $d$-band edge (just below the Fermi level $E_{F}$ ), there is a reduction in the DOS for $\mathrm{H}$ in tetrahedral and surface threefold sites corresponding to the quenching of some $\mathrm{Pd}$ states by $\mathrm{H}$ chemisorption. [Figs. 2(a) and 2(c)]. This reduction is less apparent for $\mathbf{H}$ in octahedral sites [Fig. 2(b)] and the DOS is closer to that of the clean surface. This feature will become more apparent in the analysis of surface band structures and the surface density of states.

\section{B. Bonding characteristics}

We now concentrate for the moment on the nature of the $\mathrm{H}-\mathrm{Pd}$ bonding band. The charge densities of this band are plotted in Fig. 3 for the three configurations. It is clear from the figure that the bonding bands are com-

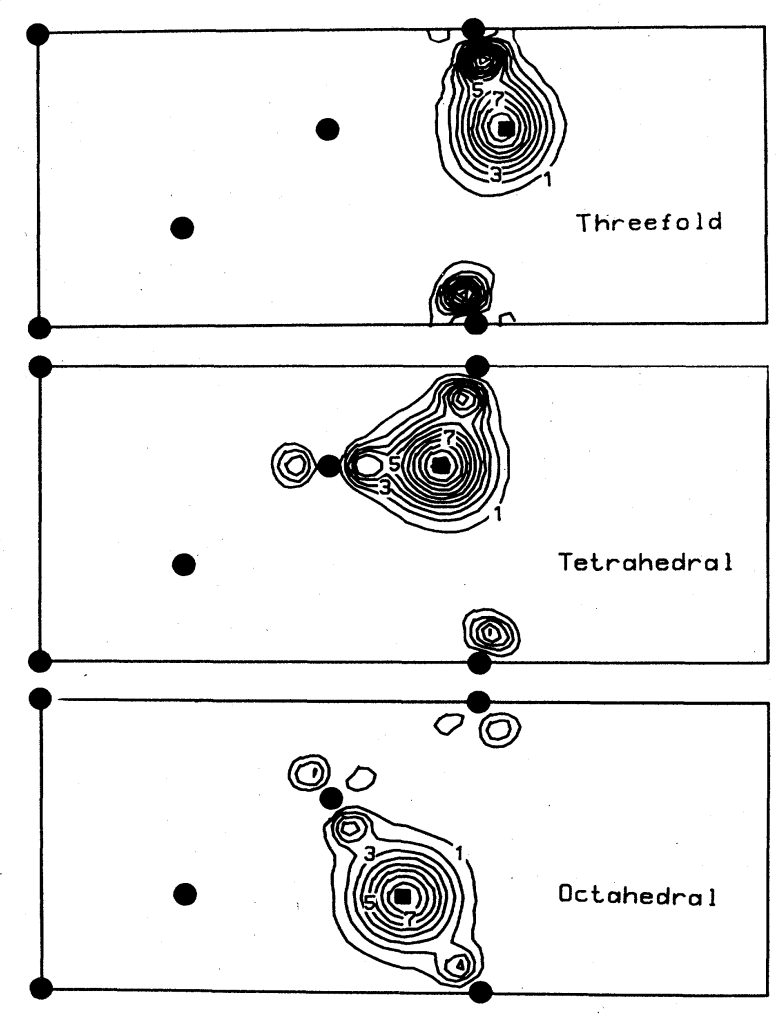

FIG. 3. Charge-density contour plots (in 0.01 electrons/a.u. ${ }^{3}$ ) on a (110) plane for the $\mathbf{P d}-\mathbf{H}$ bonding band with $\mathbf{H}$ at various sites. Circles indicate $\mathbf{P d}$ sites and squares indicate $\mathbf{H}$ sites. 
posed of surface states formed by the interactions between the surface $\mathbf{P d}$ atoms and $\mathrm{H}$. We have projected out the angular-momentum components of the bonding states according to

$$
C_{n, \overrightarrow{\mathrm{k}}}^{l}(\vec{\tau})=2 \sum_{m} \int_{\Omega} \psi_{n, \overrightarrow{\mathrm{k}}}^{*}(\overrightarrow{\mathrm{r}}-\vec{\tau}) \hat{P}_{l m} \psi_{n, \overrightarrow{\mathrm{k}}}(\overrightarrow{\mathrm{r}}-\vec{\tau}) d \overrightarrow{\mathrm{r}}
$$

where $l m$ are the angular momentum indices, $\hat{P}_{l m}$ is the angular-momentum projection operator, $\vec{\tau}$ is the basis vector either of a surface $\mathrm{Pd}$ site or a $\mathrm{H}$ site, $n$ is the band index, and $\Omega$ is a spherical volume of radius $R$ ( 2 a.u. for $\mathrm{Pd}$ and 1.5 a.u. for $\mathrm{H}$ ) about the atomic sites. Decomposition of the wave functions of the $\mathbf{H}-\mathbf{P d}$ bonding states around the atomic sites shows that for a general $\overrightarrow{\mathrm{k}}$ point, the bonding character is mainly $s$ like about the $\mathbf{H}$ site and mainly $d$ like about the Pd site. Thus the bonding is $\mathrm{H} 1 s-\mathrm{Pd} 4 d$, as in the case of Pd hydride. The decompositions for several $\overrightarrow{\mathrm{k}}$ points are listed in Table I. For $\mathbf{H}$ on surface threefold sites, only the angular-momentum components for the Pd site at the top layer are listed, since the bonding is completely localized on the surface layer. For $\mathbf{H}$ in subsurface sites, the bonding band wave function is spread over the top two layers and so angular-momentum components for both the top layer and second layer are tabulated. Note that for all the $\overrightarrow{\mathrm{k}}$ points listed, the $s$ and $p$ components at the $\mathrm{Pd}$ sites with $\mathrm{H}$ at the surface threefold sites are approximately equal to those of the subsurface sites (with the contributions from the top two layers added together). On the other hand, the $d$ components at or near $\bar{\Gamma}$ are very sensitive to the site of $\mathbf{H}$ chemisorption. At $\overrightarrow{\mathrm{k}}=(0,0)$ and $\overrightarrow{\mathrm{k}}=\left(\frac{1}{12}, \frac{1}{12}\right)$, the $d$ components at the $\mathrm{Pd}$ sites with $\mathrm{H}$ at subsurface octahedral sites are significantly smaller than those with $\mathbf{H}$ at subsurface tetrahedral sites or surface threefold sites. At the $\mathbf{H}$ site, the character of the $\mathbf{H}-\mathbf{P d}$ bonding states for all cases are essentially $s$ like as expected.

Integration of the charge density in the bonding band shows that the $\mathbf{H}$ atom in all cases has more charge within a given radius than the corresponding free neutral $\mathbf{H}$ atom. This is consistent with the higher electronegativity of $\mathrm{H}$. This is also consistent with an increase in the work function of the $\mathrm{Pd}(111)$ surface upon chemisorption of $\mathbf{H}$ (Refs. 7 and 20) and the small chemical shift of Pd core levels towards higher binding energy observed in the bulk hydride. ${ }^{9}$

TABLE I. Angular-momentum components (in units of number of electrons) of the Pd-H bonding band for selected $\vec{k}$ points. The $\overrightarrow{\mathrm{k}}$ points are given in units of the basis vectors of the 2D BZ. For the subsurface sites, the bonding band wave function is spread over the top two layers and decompositions for Pd sites in both layers are listed. Top-layer Pd sites are indicated by (1) and the second layer by (2). Note the very small $d$ components for $\overrightarrow{\mathrm{k}}$ points $(0,0)$ and $\left(\frac{1}{12}, \frac{1}{12}\right)$ for $\mathrm{H}$ in octahedral sites. The relative positions of various $\overrightarrow{\mathrm{k}}$ points are indicated in Fig. 7 .

\begin{tabular}{|c|c|c|c|c|c|c|}
\hline \multicolumn{4}{|c|}{$\mathbf{P d}$} & \multicolumn{3}{|c|}{$\mathbf{H}$} \\
\hline & $s$ & $p$ & $d$ & $s$ & $p$ & $d$ \\
\hline \multicolumn{7}{|l|}{$\overrightarrow{\mathbf{k}}=(0,0)$} \\
\hline Threefold & 0.35 & 0.0 & 0.25 & 0.58 & 0.01 & 0.0 \\
\hline \multirow[t]{2}{*}{ Octahedral } & (1) 0.16 & 0.02 & 0.0 & 0.6 & 0.0 & 0.0 \\
\hline & (2) 0.19 & 0.01 & 0.0 & & & \\
\hline \multirow[t]{2}{*}{ Tetrahedral } & (1) 0.28 & 0.01 & 0.14 & 0.7 & 0.0 & 0.0 \\
\hline & (2) 0.05 & 0.02 & 0.07 & & & \\
\hline \multicolumn{7}{|l|}{$\overrightarrow{\mathrm{k}}=\left(\frac{1}{4}, \frac{1}{4}\right)$} \\
\hline Threefold & 0.02 & 0.06 & 0.82 & 0.72 & 0.01 & 0.0 \\
\hline \multirow{2}{*}{ Octahedral } & (1) 0.02 & 0.03 & 0.30 & 0.64 & 0.0 & 0.0 \\
\hline & (2) 0.02 & 0.03 & 0.33 & & & \\
\hline \multirow[t]{2}{*}{ Tetrahedral } & (1) 0.02 & 0.06 & 0.54 & 0.82 & 0.0 & 0.0 \\
\hline & (2) 0.03 & 0.01 & 0.15 & & & \\
\hline \multicolumn{7}{|l|}{$\overrightarrow{\mathbf{k}}=\left(\frac{1}{3}, \frac{1}{12}\right)$} \\
\hline Threefold & 0.06 & 0.04 & 0.84 & 0.64 & 0.02 & 0.0 \\
\hline \multirow[t]{2}{*}{ Octahedral } & (1) 0.03 & 0.03 & 0.27 & 0.63 & 0.0 & 0.0 \\
\hline & (2) 0.04 & 0.03 & 0.30 & & & \\
\hline \multirow[t]{2}{*}{ Tetrahedral } & (1) 0.05 & 0.05 & 0.53 & 0.79 & 0.0 & 0.0 \\
\hline & (2) 0.03 & 0.01 & 0.14 & & & \\
\hline \multicolumn{7}{|l|}{$\overrightarrow{\mathrm{k}}=\left(\frac{1}{12}, \frac{1}{12}\right)$} \\
\hline Threefold & 0.3 & 0.03 & 0.29 & 0.63 & 0.01 & 0.0 \\
\hline \multirow[t]{2}{*}{ Octahedral } & (1) 0.14 & 0.03 & 0.03 & 0.64 & 0.0 & 0.0 \\
\hline & (2) 0.16 & 0.03 & 0.03 & & & \\
\hline \multirow[t]{2}{*}{ Tetrahedral } & (1) 0.24 & 0.03 & 0.17 & 0.74 & 0.0 & 0.0 \\
\hline & (2) 0.04 & 0.02 & 0.09 & & & \\
\hline
\end{tabular}


Near $\bar{\Gamma}$, the center of the 2D BZ, the bonding for the split-off band is between $\mathrm{H} 1 s$ and Pd states of $\Lambda_{1}$ symmetry $\left(s\right.$ and $d_{3 z^{2}-r^{2}}$, with $z$ axis along the normal of the surface). This is imposed by the $C_{3 v}$ symmetry of the (111) surface. As shown in Table I the bonding state has virtually no $d$ character at the $\mathrm{Pd}$ site for $\mathrm{H}$ at the octahedral sites, whereas for the other sites, the bonding state has both $s$ - and $d$-like $\left(d_{3 z^{2}-r^{2}}\right)$ components. This feature can also be observed from Fig. 4, where we have plotted the charge densities of the $\mathbf{H}-\mathbf{P d}$ bonding band at $\bar{\Gamma}$. This lack of $d$ character is a consequence of the $C_{3 v}$ symmetry of the (111) surface and the angular position of the octahedral sites. ${ }^{21}$ Under $C_{3 v}$ symmetry, the states at $\bar{\Gamma}$ must be of either $\Lambda_{1}$ or $\Lambda_{3}$ symmetry. The H $1 s$ orbitals interact with Pd states which have $\Lambda_{1}$ symmetry to form a bonding state. States of $\Lambda_{1}$ symmetry can only be composed of $s, p_{z}$, and $d_{3 z^{2}-r^{2}}$ orbitals on the surface Pd atoms. It happened that $\mathrm{H}$ at the octahedral sites is in the nodal plane of the $d_{3 z^{2}-r^{2}}$ orbitals of the Pd atoms, and so there is no nearest-neighbor interaction between these orbitals. In more concrete terms, we can use a LCAO description (in Slater-Koster notation). The interatomic matrix element between a $d_{3 z^{2}-r^{2}}$ orbital and a $s$ orbital at an angle $\theta$ with respect to the $z$ axis can be expressed as

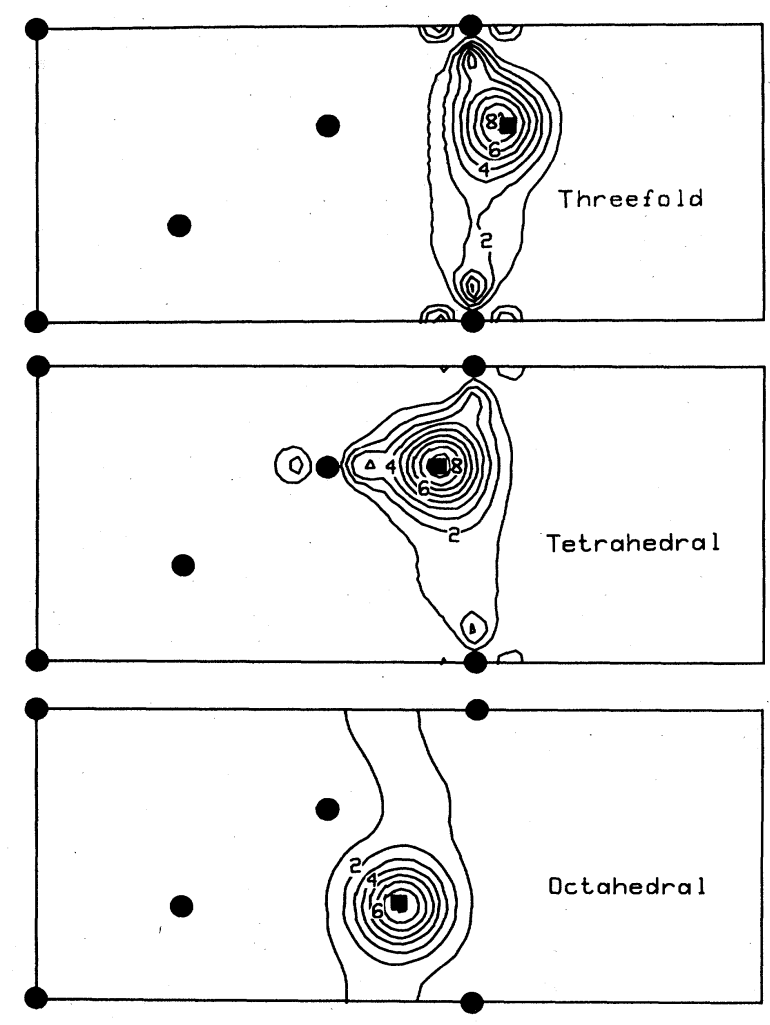

FIG. 4. Charge-density contour plots (in 0.01 electrons/a.u. ${ }^{3}$ ) on a (110) plane for the $\mathbf{P d}-\mathbf{H}$ bonding state at $\bar{\Gamma}$ with $\mathbf{H}$ at various sites. Circles indicate $\mathbf{P d}$ sites and squares indicate $\mathbf{H}$ sites.

$$
E_{s, d}{ }_{3 z^{2}-r^{2}}=P_{2}(\cos (\theta)) V_{s d \sigma},
$$

where $P_{2}(\cos (\theta))=\frac{1}{2}\left(3 \cos ^{2} \theta-1\right)$ is just the angular part of the $d_{3 z^{2}-r^{2}}$ orbital. This is plotted in Fig. 5 for the case of $\mathrm{H}$ in the position of the octahedral site. We note that $\mathrm{H}$ at the octahedral sites are at $\theta=\cos ^{-1}(1 / \sqrt{3})$ which would make Eq. (2) identically zero. Hence at $\bar{\Gamma}$, the $\mathrm{Pd} d$ orbitals cannot interact with the $\mathrm{H} 1 s$ orbitals through nearest-neighbor interactions (within two-center approximations) and the $d$ states do not mix into the H-Pd bonding state. For other sites, the interatomic matrix element between $\mathrm{H} 1 s$ and $\mathrm{Pd} d_{3 z^{2}-r^{2}}$ orbitals is nonzero and hence a substantial amount of $d$ characters are in the bonding state. Since $s$-like states have smaller photoemission cross sections than $d$-like states, ${ }^{13}$ this result suggests that it may be difficult to detect the split-off bonding states for $\mathrm{H}$ at octahedral sites by normalemission experiments which only sample states near the $\bar{\Gamma}$ point. Another feature that distinguishes the subsurface octahedral sites from the other sites is that the bonding state is more delocalized.

For the outer part of the 2D BZ, our results show that the bonding is mainly between the $\operatorname{Pd} x^{2}-y^{2}, x y, x z$, and $y z 4 d$ orbitals with the $\mathrm{H} 1 s$ orbital. This is true for all the sites we have considered. The Pd-H bonding band should hence be detectable in off-normal angle-resolved photoemission measurements for $\mathbf{H}$ in the subsurface sites since this band has been observed for $\mathbf{H}$ on the surface threefold sites. As the outer part of the $2 \mathrm{D}$ BZ is dominant over the region near $\bar{\Gamma}$, the overall bonding characteristics are expected to be rather similar in all cases. Thus, an angle-integrated photoemission experiment should show a $\mathbf{H}$-induced peak for all three cases except for a difference in intensity and its position relative to the Pd $d$ bands. However, an angle-resolved normal emission experiment will have distinctly different results for $\mathbf{H}$ at the octahedral sites due to the change in bonding characteristics at $\bar{\Gamma}$.

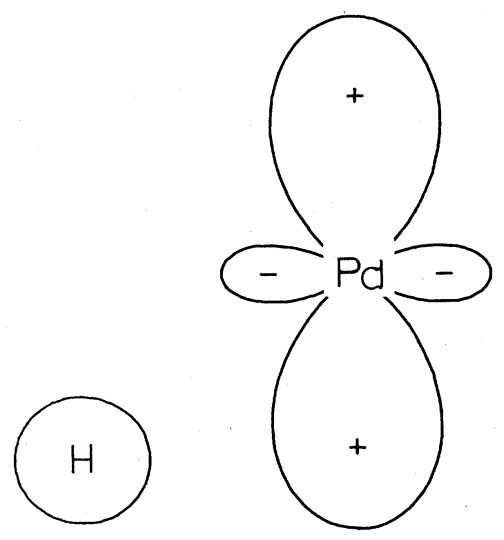

FIG. 5. Schematic plot of the position of a surface Pd $d_{3 z^{2}-r^{2}}$ orbital with respect to a $\mathbf{H} 1 s$ orbital at an octahedral site. 


\section{Surface states and projected band structure}

The projected band structure and the surface states along the high-symmetry lines $\bar{\Sigma}$ and $\bar{T}$ for $\mathrm{H}$ in subsurface octahedral and tetrahedral sites are shown in Figs. 6(a) and 6(b), respectively. Surface states are marked by solid lines and strong surface resonances by dashed lines. The shaded areas are the allowed bulk states. Surface band structures for the clean $\mathrm{Pd}(111)$ surface and with $\mathbf{H}$ chemisorbed in surface threefold sites have been reported. ${ }^{2}$ They are reproduced in Fig. 7 for the ease of comparison. In Fig. 7 solid lines are surface states for $\mathbf{H}$ on the threefold sites, and the dashed lines are the intrinsic surface states of the clean surface.

The common and most prominent of the $\mathrm{H}$-induced features in the chemisorbed surface band structure is the
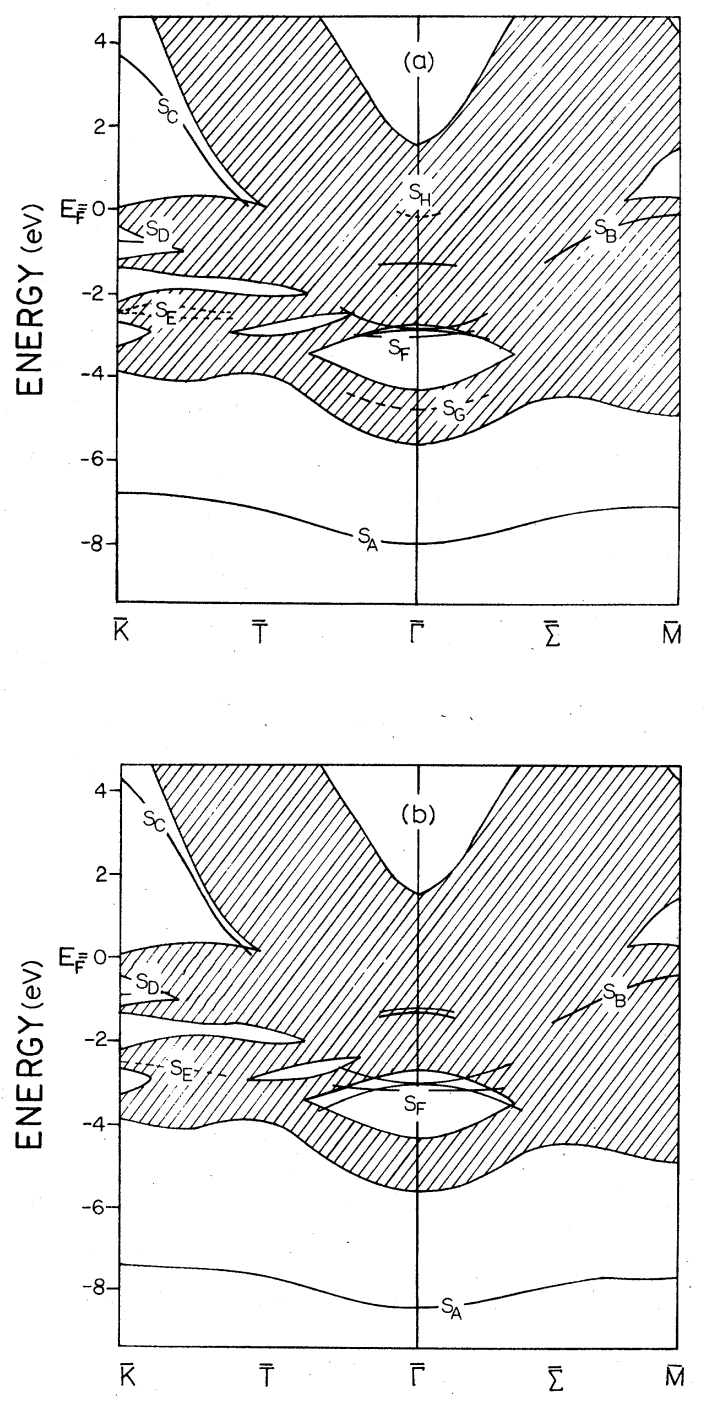

FIG. 6. Surface states and resonances for $\mathrm{H}$ in (a) subsurface octahedral sites, and (b) subsurface tetrahedral sites. Solid lines are the surface states and dashed lines are strong surface resonances. The shaded areas are the projection of the Pd bulk bands onto the (111) surface.

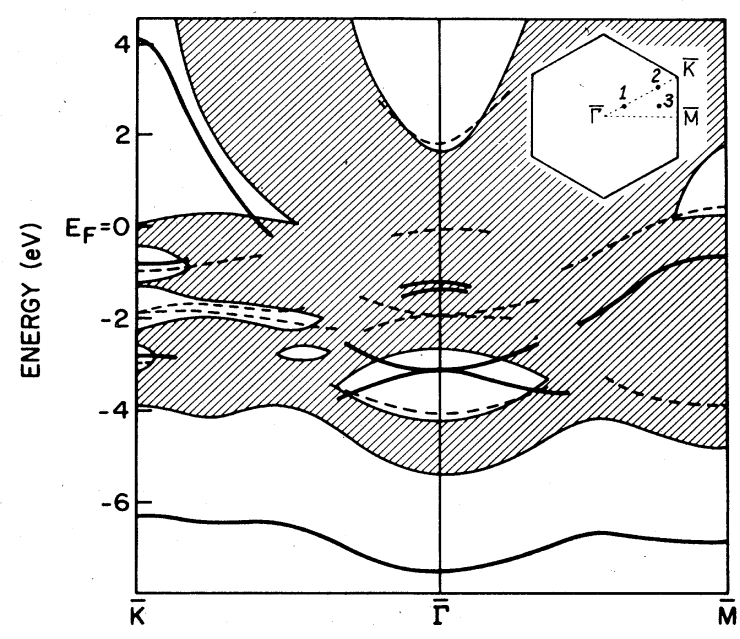

FIG. 7. Surface states and resonances for the clean Pd(111) surface (dashed lines) and $\mathrm{H}$-chemisorbed surface with $\mathrm{H}$ in the surface threefold site (solid lines). (From Ref. 2.) The twodimensional Brillouin zone is shown in the inset. The irreducible zone is indicated by dashed lines. The points labeled 1,2,3 are the $\overrightarrow{\mathrm{k}}$ points $\left(\frac{1}{12}, \frac{1}{12}\right),\left(\frac{1}{4}, \frac{1}{4}\right)$, and $\left(\frac{1}{3}, \frac{1}{12}\right)$ as quoted in Table I.

existence of a split-off $\mathrm{Pd}-\mathrm{H}$ bonding band (labeled as $S_{A}$ in Fig. 6) extending over the entire 2D BZ. This band, as discussed in Sec. II A, is lowest in energy for $\mathrm{H}$ in the tetrahedral sites and highest for $\mathbf{H}$ in surface threefold sites. Near the zone boundaries (near $\bar{K}$ and $\bar{M}$ ), the qualitative features of the surface states for $\mathbf{H}$ occupying different sites are similar. For example, for both the surface and subsurface chemisorption sites considered, there is a $\bar{\Sigma}_{2}$ surface resonance $\left(S_{B}\right.$ in Fig. 6 ), which is odd with respect to a vertical plane containing $\bar{\Gamma} \bar{M}$. It starts at $\bar{M}$ at slightly below $E_{F}$ as a $\bar{M}_{2}$ surface resonance and disperses downwards to approximately halfway from $\bar{M}$ to $\bar{\Gamma}$. This surface-resonance band originates from the lowering in energy of a surface band on the clean surface (Fig. 7) of the same symmetry and dispersion that is just above $E_{F}$ and exists in the energy gap at $\bar{M}$. The $\bar{\Sigma}_{1}$ surface resonance in the clean surface which terminates at $\bar{M}$ at slightly above $-4 \mathrm{eV}$ disappears upon chemisorption. In the $\bar{T}$ direction, there is an unoccupied surface band $\left(S_{c}\right)$ above $E_{F}$. It starts at $\bar{K}$ as a $\bar{K}_{2}$ surface state at $\sim 4.1 \mathrm{eV}$ for $\mathrm{H}$ in threefold sites, $\sim 3.7 \mathrm{eV}$ in subsurface octahedral sites, and $\sim 4.2 \mathrm{eV}$ in subsurface tetrahedral sites, and disperses downwards until it meets the bulk band continuum. This is an antibonding band as a result of Pd-H interaction. For both the subsurface sites there is a surface band $\left(S_{D}\right)$ in the first energy gap below $E_{F}$ near $\bar{K}$ which has $\bar{K}_{1}$ symmetry at $\bar{K}$. A similar feature has been found for the case of $H$ in surface threefold sites both theoretically and experimentally. ${ }^{7}$ The pair of intrinsic surface bands that terminates at $\bar{K}$ at $\sim-2 \mathrm{eV}$ with $\bar{K}_{2}$ symmetry on the clean surface is removed from the gap under chemisorption. They remain as surface resonances at slightly lower energies for $H$ in subsurface sites and are indicated by dashed lines $\left(S_{E}\right)$ in Fig. 6 . 
It is at the $2 \mathrm{D} \mathrm{BZ}$ center that effects of the specific position of the chemisorption site upon the surface states become more apparent. A comparison of Figs. 6(a) and 7 shows that the qualitative features of the surface states for $H$ in the octahedral sites near $\bar{\Gamma}$ resemble those of the clean surface. Near $\bar{\Gamma}$, a correspondence can be identified between the intrinsic surface states on the clean surface and the surface states for $\mathrm{H}$ in subsurface octahedral sites. The presence of $\mathrm{H}$ in subsurface octahedral sites appears to just lower the energy of the intrinsic surface states near $\bar{\Gamma}$. On the other hand, the surface states for $\mathbf{H}$ in tetrahedral sites resemble closely those of $\mathbf{H}$ in surface threefold sites. The only additional feature that is specific to subsurface geometries is the existence of an additional surface state $\left(S_{F}\right)$ with $\Lambda_{1}$ symmetry at $\bar{\Gamma}$ near $-3 \mathrm{eV}$ just below a set of $\Lambda_{3}$ surface states at $\bar{\Gamma}$ for $H$ in both the subsurface sites. This is a "subsurface feature" since both the clean surface and the $H$ in surface threefold sites do not have this surface state. We note that there is a big absolute gap in the projected band structure at $\sim-2.7$ to $\sim-4.4 \mathrm{eV}$ at $\bar{\Gamma}$, but this gap is almost closed in the stoichiometric bulk hydride. This can be seen by examining published band structure of bulk $\mathrm{PdH}$ from $\Gamma$ to $L{ }^{12}$ The occupation of subsurface sites by $\mathrm{H}$ can be regarded as the formation of a hydride in the surface layers, so it is not surprising to see surface bands splitting off into an energy region forbidden in the pure $\mathrm{Pd}$ but allowed in the bulk hydride.

The resemblance of the surface features for $\mathrm{H}$ in octahedral sites to those of the clean surface can be interpreted as follows. As shown in Fig. 7, the clean surface has four sets of surface states near $\bar{\Gamma}$. The surface states at $\sim 2$ and $\sim-4 \mathrm{eV}$ have $\Lambda_{1}$ symmetry at $\bar{\Gamma}$, while the surface states at $\sim 0$ and $\sim-2 \mathrm{eV}$ have $\Lambda_{3}$ symmetry at $\bar{\Gamma}$. Upon chemisorption of $\mathbf{H}$ at surface threefold sites or subsurface tetrahedral sites, the $\Lambda_{1}$ surface at $\sim-4 \mathrm{eV}$ interacts with $\mathrm{H}$ to form the split-off bonding band. The $\Lambda_{3}$ surface states at or near $\bar{\Gamma}$ do not couple to the $H$ directly and they are not destroyed by chemisorption. The $\Lambda_{3}$ surface states are, however, lowered in energy because of the presence of $\mathrm{H}$. The surface state at $2 \mathrm{eV}$ disappears as it merges into the bulk band manifold for $\mathrm{H}$ in threefold and subsurface tetrahedral sites. The similarity of the surface features for surface threefold and subsurface tetrahedral sites is a consequence of the similarity in local bonding environment, as discussed above.

However, for $\mathbf{H}$ in the octahedral sites, the symmetry forbids two-center nearest-neighbor interactions between the $\mathrm{Pd} d_{3 z^{2}-r^{2}}$ orbitals with the $\mathrm{H} 1 s$ orbitals. As a consequence, the $d$-like part of $\Lambda_{1}$ surface states is not completely destroyed upon chemisorption. Their energy is lowered and they survive as resonances in the bulk band region with considerable amplitude near the top two layers. These states are indicated as dashed lines $\left(S_{H}\right.$ and $S_{G}$ ) in Fig. 6(a). They have $\Lambda_{1}$ symmetry at $\bar{\Gamma}$. The surface resonance $S_{H}$ has considerable $s p$ character and is similar to the $\Lambda_{1}$ surface state at $2 \mathrm{eV}$ of the clean surface, while $S_{G}$ has considerable $d_{3 z^{2}-r^{2}}$ character and is similar to the $\Lambda_{1}$ surface state at $\sim-4 \mathrm{eV}$ of the clean surface. The symmetry arguments are valid only at $\bar{\Gamma}$. Away from the zone center, the surface features are not as sensitive to the site of $\mathrm{H}$.

\section{Local density of states}

We have calculated the layerwise $\overrightarrow{\mathrm{k}}$-vector-resolved local density of states (LDOS) defined as

$$
D_{L}(\epsilon, \overrightarrow{\mathrm{k}})=2 \sum_{n} \delta\left(\epsilon-\epsilon_{n, \overrightarrow{\mathrm{k}}}\right) \int_{\Omega_{L}}\left|\psi_{n, \overrightarrow{\mathrm{k}}}\right|^{2} d \overrightarrow{\mathrm{r}},
$$

where $\Omega_{L}$ is the volume of the $L$ th layer in the slab. The outermost layer is referred to as the first layer. In order to facilitate a more direct comparison with experimental photoemission spectra, the LDOS for the occupied states are transformed to a model emission function by convoluting with a Gaussian broadening function such that

$$
\begin{gathered}
\bar{D}_{L}(\epsilon, \overrightarrow{\mathrm{k}})=\int_{ \pm \infty}^{E_{F}} D_{L}\left(\epsilon^{\prime}, \overrightarrow{\mathrm{k}}\right)\left[\alpha\left(\epsilon^{\prime}\right) / \pi\right]^{1 / 2} \\
\times e^{-\alpha\left(\epsilon^{\prime}\right)\left(\epsilon-\epsilon^{\prime}\right)^{2}} d \epsilon^{\prime}
\end{gathered}
$$

The exponent $\alpha(\epsilon)$ corresponds to a full width at half maximum $\gamma(\epsilon)$ given by $\gamma(\epsilon)=0.2+0.15\left|\epsilon-E_{F}\right| \mathrm{eV}$. This is to account for instrumental and lifetime broadening. ${ }^{2,7}$ A comparison of the LDOS for different local adsorption geometries layer by layer can provide us with useful information about the perturbation of $\mathbf{H}$ on the substrate. We plot in Figs. 8(a)-8(d) the LDOS for the occupied states layer by layer at $\bar{K}$ for four systems: $\operatorname{Pd}(111)$ clean surface, $H$ in subsurface octahedral and tetrahedral sites, and $\mathrm{H}$ on surface threefold sites. Figures 8(a) and 8(b) show that the LDOS for the fourth layers (center layers) and the third layers are almost identical for all the systems as expected. $\mathrm{H}$-induced variations start to develop at the second layer [Fig. 8(c)]. For the $\mathrm{H}$ chemisorbed surfaces, the peaks in the LDOS corresponding to the $\mathrm{Pd} d$ states are shifted down slightly in energy relative to the corresponding peaks of the clean surface. The split-off $\mathbf{P d}-\mathrm{H}$ bonding-state peak already appears in the second layer for $H$ in the subsurface sites. It is stronger for the octahedral than the tetrahedral sites since the $\mathrm{Pd}-\mathrm{H}$ bonding-state wave function is shared by an equal number of Pd atoms on the top and second layer for $\mathbf{H}$ in the octahedral site while the bonding state is shared by three $\mathbf{P d}$ atoms on the surface and only one atom in the second layer for $\mathrm{H}$ at the tetrahedral site. The bonding state wave function for $\mathrm{H}$ on surface threefold sites is solely confined to the first layer. At the surface layer [Fig. 8(d)], the $\mathrm{H}$-induced features are fully developed. A well-defined $\mathrm{H}$-induced state splits off from the $d$ bands with energy position dependent on the chemisorption site. Also, the shifting in the energy position of the Pd $d$-state peaks appears to be larger than the shifting in the second layer.

The occupied surface DOS (SDOS) which is defined to be the LDOS (of the occupied states) for the top two layers, and the vacuum, are plotted in Figs. 9-11 for $k$ points $\bar{M}, \bar{K}$, and $\bar{\Gamma}$, respectively. The SDOS is smoothed the same way as the LDOS except that the constant term in the empirical expressions for the full width at half maximum is increased from 0.2 to $0.4 \mathrm{eV}$. This gives a 


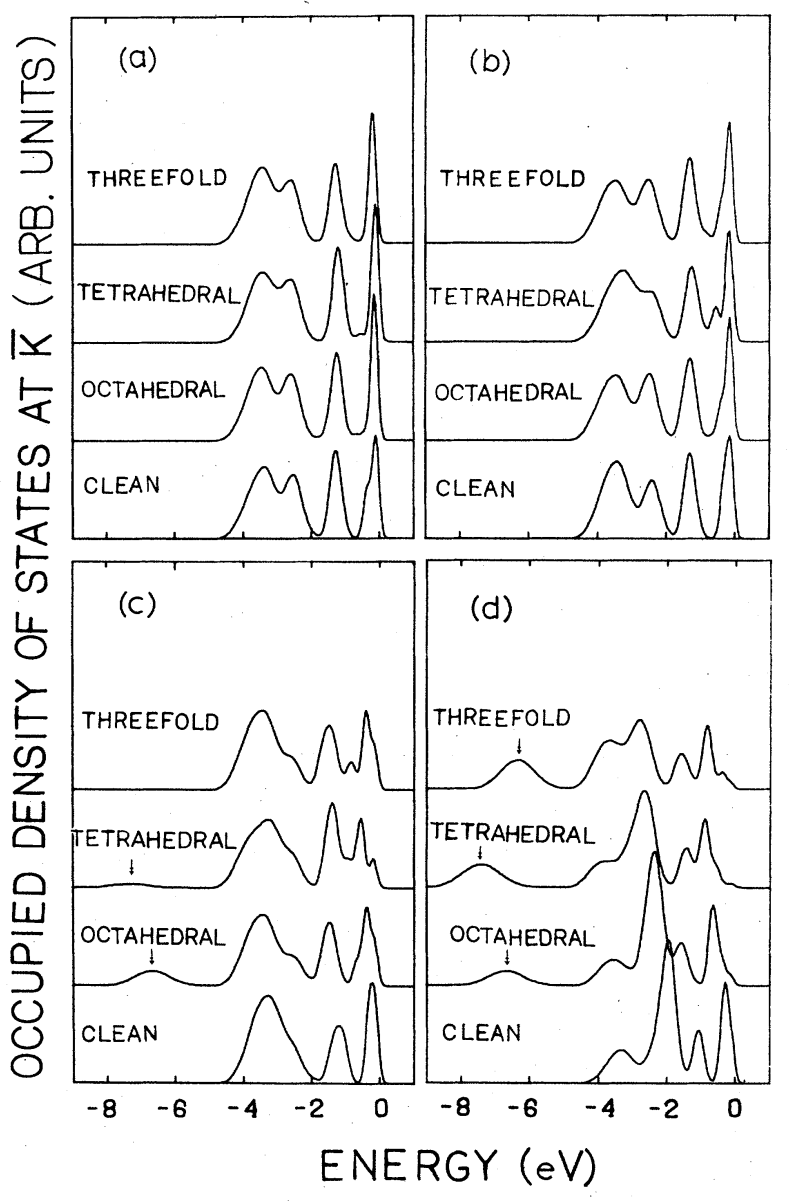

FIG. 8. Layerwise LDOS for the occupied states at $\bar{K}$. (a) is the fourth (middle) layer, (b) is the third layer, (c) is the second layer, and (d) is the first (surface) layer. The peaks due to $\mathrm{H}$ induced bonding states are indicated by arrows.

better simulation of the energy resolution of the experimental ${ }^{7}$ spectra for surface threefold sites.

The occupied SDOS at $\bar{M}$ (Fig. 9) for the three chemisorbed system are rather similar except for the position of the Pd-H bonding state (indicated by arrows). At $\bar{K}$ (Fig. $10)$, the $d$-band region of the SDOS for $\mathrm{H}$ in the octahedral site bears more resemblance to the clean surface than the other two chemisorption sites considered. The same is true at $\bar{\Gamma}$ (Fig. 11). It would thus be more difficult to detect subsurface octahedral $\mathbf{H}$ by just examining the $d$ band region of the photoemission spectra. Likewise, it would be difficult to distinguish between surface threefold sites from subsurface tetrahedral sites by comparing the $d$-band spectra.

The occupied SDOS at $\bar{\Gamma}$ for $\mathrm{H}$ at octahedral site resembles that of the clean surface closely partly because the downward shift in energy of the $d$-band states upon chemisorption is smallest for the octahedral site and partly because of the existence of a $\Lambda_{1}$ surface resonance near $E_{F}$. This resemblance of the SDOS to the clean surface in the $d$-band region, together with the lack of the $d$ character in the bonding state, may cause the normal-emission

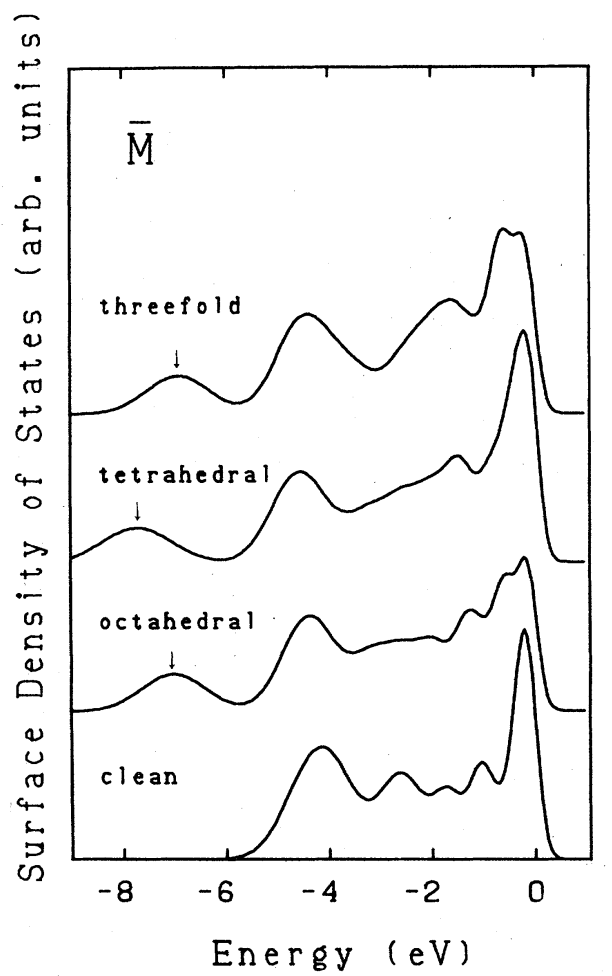

FIG. 9. SDOS for the occupied states at $\bar{M}$. The peaks due to the $\mathrm{H}$-induced bonding states are indicated by arrows.

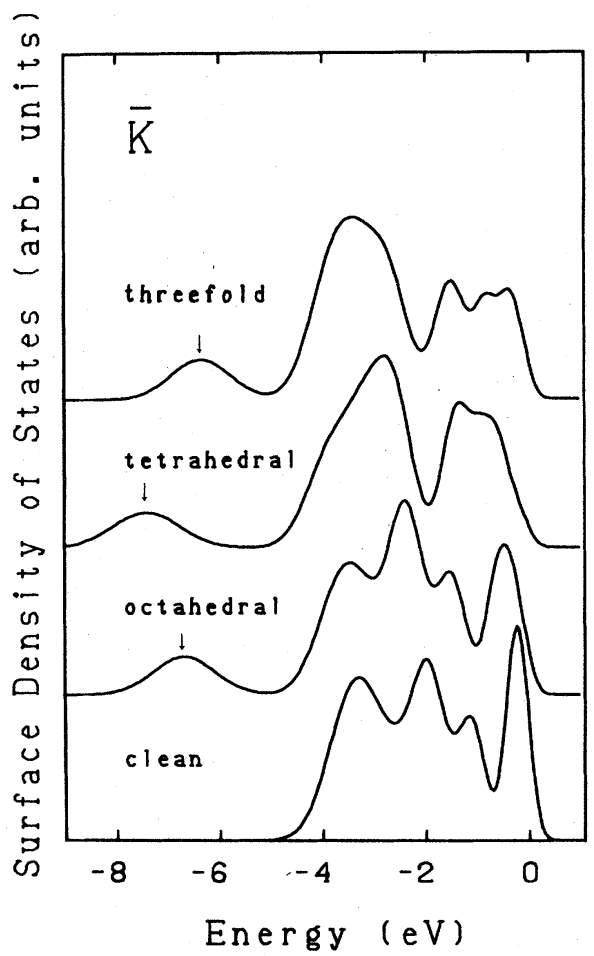

FIG. 10. SDOS for the occupied states at $\bar{K}$. The peaks due to the $\mathbf{H}$-induced bonding states are indicated by arrows. 


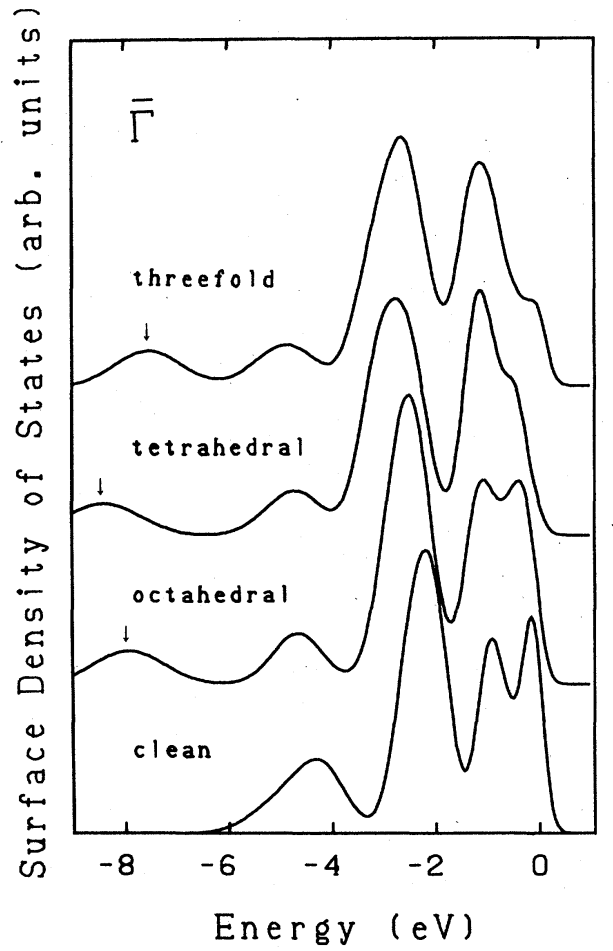

FIG. 11. SDOS for the occupied states at $\bar{\Gamma}$. The peaks due to the $\mathbf{H}$-induced bonding states are indicated by arrows.

spectra of $\mathrm{H}$ at the subsurface octahedral site to be very similar to that of a clean surface. However, even at normal emission one can in principle distinguish the clean (111) surface from one with $\mathrm{H}$ chemisorbed at the subsurface octahedral site if the experiment is performed with polarized light. Figure 12 shows the SDOS at $\bar{\Gamma}$ decomposed into $\Lambda_{1}$ and $\Lambda_{3}$ components. There is obviously less resemblance between the clean surface and $\mathrm{H}$ at octahedral site after decomposition of SDOS into symmetry components. Since $\Lambda_{1}$ states can only be excited by $p$ -
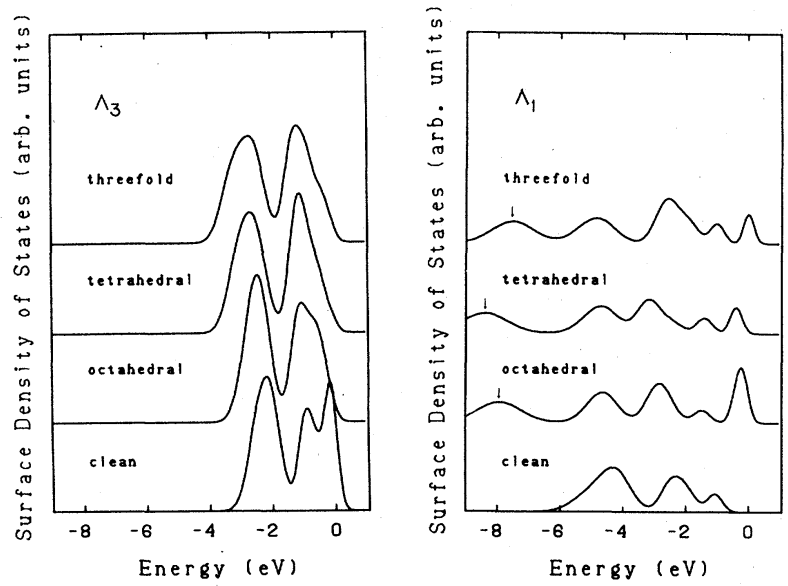

FIG. 12. SDOS for the occupied states at $\bar{\Gamma}$ decomposed into symmetry components. The peaks due to $\mathrm{H}$-induced bonding states are indicated by arrows. polarized light while $\Lambda_{3}$ states can only be excited by $s$ polarized light (within the dipole approximation), polarization-dependent normal-emission ultraviolet photoemission spectroscopy can establish more unambiguously whether the subsurface octahedral sites are occupied.

\section{DISCUSSION}

In our present calculations, we have assumed a $(1 \times 1)$ ordered layer of $\mathrm{H}$. We note in Figs. 6 and 7 that most of the surface states and resonances of the clean surface, except for those participated in direct bonding to $\mathrm{H}$, survive upon the addition of a layer of $\mathrm{H}$. All these surface features have substantial amplitudes on the surface. This is a direct consequence of the $(1 \times 1)$ chemisorption geometry used in the present calculations. Had we adopted a lower symmetry (or assumed randomness) for the $\mathbf{H}$ layer, the intrinsic surface states and resonances would have broadened substantially by the argument of Kar and Soven. ${ }^{22}$ This is because the lowering of symmetry would fold the 2D BZ back into itself, as a result of the reduction of the size of the irreducible zone. This mechanism would broaden surface states or resonances. Experimentally, it was found that most of the surface states on $\operatorname{Pd}(111)$ persist upon chemisorption at low temperature, ${ }^{7}$ consistent with a $(1 \times 1)$ ordered arrangement of the $\mathrm{H}$. Further evidence comes from the $\mathbf{P d}-\mathbf{H}$ bonding band, which has a dispersion the same as that required by a $(1 \times 1)$ symmetry. ${ }^{7}$ The agreement of the positions and dispersions of the calculated surface states for $\mathbf{H}$ in the threefold surface sites with those determined from lowtemperature experiments ${ }^{7}$ justifies other assumptions used in the present calculations. For the high-temperature phase, we cannot tell the symmetry of the chemisorbed system from the Pd-H bonding band, as it is not observed. Normal-emission experiments indicate the surface states at $\bar{\Gamma}$ persist in the high-temperature phase. ${ }^{6,7}$ There is no observable broadening compared with the cleansurface spectra. ${ }^{7}$ If $\mathbf{H}$ are indeed present at a coverage up to $\sim \frac{1}{2}$ monolayer ${ }^{6,7}$ in the high-temperature phase, they should have a $(1 \times 1)$ ordered arrangement (may be in the form of islands) in order to reconcile with the nonbroadening of the surface states. In that case, the present $(1 \times 1)$ ordered monolayer subsurface calculations may be used to interpret the high-temperature spectra.

A recent effective-medium calculation of Puska et al. ${ }^{23}$ indicates strong "quantum motion" for $\mathrm{H}$ adatoms on Ni surfaces. Their results of $\mathrm{H}$ on $\mathrm{Ni}(100)$ and $\mathrm{Ni}(111)$ show that the ground state for the " $\mathrm{H}$ band" (describing the motion of the $\mathrm{H}$ atoms) is dispersionless, implying that the $\mathrm{H}$ are well localized in their chemisorption sites. Thus at low temperatures (which means occupation of ground state) and close to full monolayer coverage (which further reduces dispersion), the classical picture of assuming $\mathrm{H}$ occupying well-defined sites is justified. The excellent comparison between the lowtemperature experimental spectra and the theoretical results for $\mathrm{H}$ on the surface threefold sites of $\mathrm{Pd}(111)$ gives us further confidence in this aspect. However, the calculations of Puska et al. indicated that excited states for atomic motion of $\mathrm{H}$ on $\mathrm{Ni}$ surface have considerable 
dispersion, especially for the (111) face. This corresponds to delocalized $\mathbf{H}$ atoms on the surface. As the excitation energy is of the order $\sim 50 \mathrm{meV}$ (Table I of Ref. 23), chemisorption at room temperature (thermal energy, $\sim 25$ $\mathrm{meV}$ ) can correspond to the occupation of delocalized $\mathbf{H}$ states. The quantum effects will be important if the coverage is low. In this case, it is better to consider the $\mathbf{H}$ atoms as forming a delocalized surface state of hydrogen atoms rather than $\mathrm{H}$ atoms occupying some specific discrete sites. It is then doubtful that the resulting photoemission spectra can be interpreted by a straightforward comparison with theoretical calculations that assume ordered monolayer adsorbate geometry. The delocalization of the adsorbates might lead to a broadening of the energy of the electronic bonding state. The effect on other metal surface states is unclear. The above theoretical results are, of course, only for $\mathrm{Ni}$. The role of $d$ electrons are much more important in $\mathrm{Pd}-\mathrm{H}$ bond than in $\mathrm{Ni}-\mathrm{H}$ bond, and the $d$ electrons are expected to produce more localized bondings.

Models like the subsurface models ${ }^{6}$ assume a temperature-induced change in chemisorption geometries. This is plausible if the energy barrier from one local minimum to another at different high-symmetry sites are of the order of the thermal energy. Experimental results of Christmann et $a .^{24}$ showed that this is probably the case for $\mathrm{Ni}(111)$. Our numerical results and the interpretation based on symmetry show that the octahedral subsurface model is consistent with normal-emission spectra of the high-temperature phase of $\mathbf{H}$ on $\operatorname{Pd}(111)$. However, the theory also predicts that the bonding states should be observable in off-normal photoemission experiments, but this was not observed in recent experiments. ${ }^{25}$ It is also interesting to see whether the subsurface model can be applied to similar systems. For $\mathrm{H}$ on $\mathrm{Cu}(111)$ (Ref. 7) and $\mathrm{Ni}(111)$ (Refs. 6 and 26), the angle-resolved photoemission results are qualitatively similar to that of $\operatorname{Pd}(111)$. The metal-H bonding state is observed as a split-off state at low temperatures but disappears at high temperatures. However, it is found that the $\Lambda_{1}$ surface states at $\bar{\Gamma}$ are quenched and the bonding states are not observed near the zone boundary for the high-temperature phase. ${ }^{26}$ This is not consistent with the subsurface model if we assume that $3 d$ transition metals behave similarly as $4 d$ Pd.

We now turn to the question as to whether the octahedral site is a favorable site for $\mathrm{H}$ chemisorption. At low temperature, surface threefold sites are initially favored as shown by the angle-resolved photoemission data. Migrating from surface sites to the bulk or subsurface sites would increase the coordination number of $\mathrm{H}$, thus increasing the number of $\mathrm{Pd}-\mathrm{H}$ bonds. However, this gain may be offset by the energy needed in expanding the lattice. The measured bulk heat of solution is actually smaller than the heat of adsorption for group-VIII metals. The heat of adsorption goes in the order $E_{\mathrm{Ni}}(0.5$ $\mathrm{eV})>E_{\mathrm{Pd}}(0.46 \mathrm{eV})>E_{\mathrm{Pt}}\left(\sim 0.3 \mathrm{eV},{ }^{6}\right)$ with only Pd forming a stable hydride (with heat of solution $0.2 \mathrm{eV}$ ). This is related to the fact that $\mathbf{P d}$ is the softest (with the smallest bulk modulus) and therefore requires the least energy in lattice expansion. Since the top layer of an ideal surface usually costs less energy to expand, it is plausible for $\mathbf{H}$ to overcome a surface barrier and migrate to subsurface sites at higher temperatures to form more bonds with Pd.

Subsurface chemisorption on transition-metal surfaces has been observed. Shih et al. ${ }^{27}$ have shown, using LEED, that $N$ occupies subsurface sites in $T i(0001)$ $\mathrm{N}(1 \times 1)$. Thermal-activated transitions of chemisorbed $\mathbf{H}$ to subsurface sites have been observed for $\mathrm{Pd}(110){ }^{28}$ For $3 d$ transition metals such as $\mathrm{Ti}$ and $\mathrm{Ni}$, there are theoretical calculations ${ }^{3,29}$ which, however, indicate that surface sites are more stable than subsurface sites. For the present system, the problem of the favorable chemisorption site for the high-temperature phase is an open question, since realistic total-energy calculations or conclusive experimental information are not yet available.

Finally, we have to point out that the most straightforward interpretation for the disappearance of $\mathrm{H}$-induced features in the high-temperature phase is that there is no $H$ on the surface. Eberhardt et al. ${ }^{6,7}$ conclude the existence of $\sim 0.5$ monolayer of $\mathrm{H}$ on $\mathrm{Pd}(111)$ based on evidence of work-function change, thermal-desorption spectra, and the intensity and shape of the secondary electron distribution. However, under similar experimental conditions, the absolute coverage of deuterium on $\mathrm{Pt}(111)$ was found to be very small by the nuclear microanalysis experiment of Norton et al. ${ }^{30}$

\section{SUMMARY}

The electronic structures and bonding characters of $\mathbf{H}$ in subsurface sites of $\mathrm{Pd}(111)$ are calculated and analyzed. A split-off $\mathrm{Pd}-\mathrm{H}$ bonding band is found for $\mathrm{H}$ in both the subsurface octahedral and tetrahedral sites and the overall bonding character is very similar to that of $\mathbf{H}$ on surface threefold sites. The energy position of the bonding band is sensitive to the chemisorption site. The electronic character near the zone center is shown to be very different for $\mathbf{H}$ in different sites. This is a consequence of symmetry requirements. The disappearance of $\mathbf{H}$-induced features in the normal-emission photoemission spectra of the high-temperature phase of $\mathbf{H}$ on $\operatorname{Pd}(111)$ bears some resemblance to $H$ occupying subsurface octahedral sites. However, there is also experimental and theoretical evidence against the subsurface model. Further experimental and theoretical efforts are needed before the situation can be clarified.

\section{ACKNOWLEDGMENTS}

This work was supported by the National Science Foundation under Grant No. DMR-83-19024 and by a program development fund from the Director of the Lawrence Berkeley Laboratory. 
${ }^{1}$ F. J. Arlinghaus, J. G. Gay, and J. R. Smith, in Theory of Chemisorption, edited by J. R. Smith (Springer, Berlin, 1980), and references therein.

${ }^{2}$ S. G. Louie, Phys. Rev. Lett. 42, 476 (1979).

${ }^{3}$ P. J. Feibelmann, D. R. Hamann, and F. J. Himpsel, Phys. Rev. B 22, 1734 (1980); P. J. Feibelmann and D. R. Hamann, ibid. 21, 1385 (1980); P. J. Feibelmann and F. J. Himpsel, ibid. 21, 1394 (1980).

${ }^{4} J$. P. Muscat and D. M. Newns, Surf. Sci. 99, 609 (1980).

${ }^{5}$ E. Wimmer, A. J. Freeman, W. Weinert, H. Krakauer, J. R. Hiskes, and A. M. Karo, Phys. Rev. Lett. 45, 1128 (1982).

${ }^{6}$ W. Eberhardt, F. Greuter, and E. W. Plummer, Phys. Rev. Lett. 46, 1085 (1981).

${ }^{7}$ W. Eberhardt, S. G. Louie, and E. W. Plummer, Phys. Rev. B 28, 465 (1983).

${ }^{8}$ F. Greuter and E. W. Plummer, Solid State Commun. 48, 37 (1983).

${ }^{9}$ D. E. Eastman, J. K. Cashion, and A. C. Switendick, Phys. Rev. Lett. 27, 35 (1971); F. Antonangeli, A. Balzarotti, A. Bianconi, E. Burattini, P. Perfetti, and N. Nistico, Phys. Lett. 55A, 309 (1975); P. A. Bennett and J. C. Fuggle, Phys. Rev. B 26, 6030 (1982); L. Schlapbach and J . P. Burger, J. Phys. Lett. 43, L273 (1982); H. Conrad, G. Ertl, J. Kuppers, and E. E. Latta, Surf. Sci. 58, 578 (1976).

${ }^{10}$ D. A. Papaconstantopoulos, B. M. Klein, J. S. Faulkner, and L. L. Boyer, Phys. Rev. B 18, 2784 (1978); J. Zbasnik and M. Mahnig, Z. Phys. B 23, 15 (1976).

${ }^{11}$ B. M. Klein and W. E. Pickett, Bull. Am. Phys. Soc. 28, 410 (1983), and unpublished.

${ }^{12}$ C. T. Chan and S. G. Louie, Phys. Rev. B 27, 3325 (1983), and references therein.
${ }^{13}$ S. M. Goldberg, C. S. Fadley, and S. Kono, J. Electron Spectrosc. Relat. Phenom. 21, 285 (1981).

${ }^{14}$ S. G. Louie, K. M. Ho, and M. L. Cohen, Phys. Rev. B 19, 1774 (1979).

${ }^{15}$ L. Hedin and B. I. Lundqvist, J. Phys. C 4, 2064 (1971).

${ }^{16}$ E. Caruthers, L. Kleinman, and G. P. Alldredge, Phys. Rev. B 9, 3330 (1974).

${ }^{17}$ S. G. Louie, Phys. Rev. Lett. 23, 1525 (1978).

18J. P. Muscat, Surf. Sci. 110, 389 (1981).

${ }^{19}$ K. Christmann, G. Ertl, and O. Schober, Surf. Sci. 40, 61 (1973).

20J. E. Demuth, Surf. Sci. 65, 369 (1977).

${ }^{21}$ C. T. Chan and S. G. Louie, Solid State Commun. 48, 417 (1983).

${ }^{22}$ N. Kar and P. Soven, Solid State Commun. 20, 977 (1976).

${ }^{23}$ M. J. Puska, R. M. Nieminen, M. Manninen, B. Chakraborty, S. Holloway, and J. K. Norskov, Phys. Rev. Lett. 51, 1081 (1983).

${ }^{24}$ K. Christmann, R. J. Behm, G. Ertl, M. A. Van Hove, and W. H. Weinberg, J. Chem. Phys. 70, 4168 (1979).

${ }^{25} \mathrm{E}$. W. Plummer (private communication).

${ }^{26}$ F. J. Himpsel, J. A. Knapp, and D. E. Eastman; Phys. Rev. B 19, 2872 (1979).

${ }^{27}$ H. D. Shih, H. Jona, D. W. Jepsen, and P. M. Marcus, Surf. Sci. 60, 445 (1976).

${ }^{28}$ K. H. Rieder, M. Baumberger, and W. Stocker, Phys. Rev. Lett. 51, 1799 (1983).

${ }^{29}$ J. K. Norskov, Phys. Rev. Lett. 48, 1620 (1982).

${ }^{30}$ P. R. Norton, J. A. Davies, and T. E. Jackman, Surf. Sci. 121, 103 (1982). 